\title{
BOLIVIA, ENTRE EL EXTRACTIVISMO Y EL BUEN VIVIR: PROPUESTAS Y ALTERNATIVAS DE LAS ORGANIZACIONES DE LA SOCIEDAD CIVIL
}

\author{
Marco Antonio Gandarillas \\ Director ejecutivo del Centro de Documentación e Información Bolivia (CEDIB)
}

DOI: $10.1387 /$ lan-harremanak. 16098

\section{ABSTRACT}

En este artículo se realiza una caracterización de la politica del gobierno boliviano de Evo Morales en lo que respecta a la modificación de la matriz productiva que se ha derivado de su accionar, objetivo irrenunciable de cualquier proceso de cambio de modelo coherente con el paradigma del Buen Vivir. Tras presentar sus insuficiencias, planteadas como estructurales e insalvables desde el propio proyecto politico que representa el Movimiento al Socialismo, se exponen una serie de propuestas alternativas.

Palabras clave: extractivismo, dependencia, matriz productiva, transición energética.

This article describes the policy of Evo Morales' government in Bolivia with regard to the changes to the productive fabric that have arisen from its actions, a nonnegotiable goal of any process of change of model coherent with the paradigm known as Buen Vivir (literally, Good Living). After outlining the weaknesses in this fabric, considered to be structural and insurmountable by the political project represented by the governing Movement for Socialism, a series of alternative proposals are presented.

Keywords: extractivism, dependence, productive fabric, energy transition. 


\section{Introducción}

Durante la última gira europea de Evo Morales (noviembre 2015), en la que destacaron los encuentros con Ángela Merkel y multinacionales como Siemens, éste declaró que su gobierno busca un acuerdo comercial con Alemania y con la Unión Europea (UE) (Agencia de Noticias Fides, 2015). Rafael Correa, otro gobernante progresista, suscribió a fines de 2014 un tratado con la UE arguyendo que con el mismo se potenciarán las exportaciones de su país (Agencia EFE, 2014). Que figuras emblemáticas del progresismo sudamericano declaren ser partidarios de los acuerdos de libre comercio o de los tratados de inversiones evidencia una transgresión ideológica y un cambio de horizonte en estos gobiernos.

La hipótesis de este trabajo es que los gobiernos progresistas apuntalan modelos económicos anclados en la exportación de materias primas, que benefician en último término a los grupos de poder multinacionales, debido a esto, se alejan de sectores populares que son afectados o se oponen a sus políticas. Atrapados por sus propias contradicciones, no aspiran a construir modelos alternativos al extractivismo y al capitalismo. En el caso boliviano, los grupos multinacionales son aliados estratégicos del gobierno de Morales, mientras que organizaciones ciudadanas y pueblos indígenas son tratados como enemigos del progreso (marcadamente capitalista) a los que se debe vencer.

En una segunda parte, esbozaremos algunos lineamientos de propuestas al modelo energético extractivista boliviano. Estas ideas se presentaron en distintos espacios con movimientos sociales bolivianos y latinoamericanos ${ }^{1}$.

\section{La crisis extractivista y el apego del gobierno de Evo a las multinacionales $^{2}$}

El Financial Times organizó en Nueva York a pedido del gobierno de Evo Morales un evento protagonizado fundamentalmente por empresas petroleras,

\footnotetext{
${ }^{1}$ A propósito de la COP 21 en Lima 2014.

2 Este acápite fue escrito con Oscar Campanini bajo el titulo Agotamiento del progresismo en Bolivia: Profundización del extractivismo y erosión de la democracia (Campanini y Gandarillas, 2015).
} 
mineras y bancos privados ${ }^{3}$. El propósito fue atraer nuevas inversiones extranjeras mostrando una nueva cara de Bolivia. Un evento de este tipo habría sido impensable hace solo una década atrás, cuando las petroleras y grupos de poder oligárquico buscaban evitar el ascenso al poder de Morales y del Movimiento al Socialismo (MAS), por una supuesta tendencia comunista.

En la misma línea, el vicepresidente Álvaro García Linera — de trayectoria izquierdista- contrajo a nombre del estado boliviano, el mayor crédito asumido por el país con China (7,5 mil millones de dólares, equivalentes al 17\% del PIB boliviano). Ese crédito tiene por finalidad un ambicioso programa de obras, pero está totalmente condicionado a que sean ejecutadas por empresas chinas.

Decisiones de este tipo disparan recuerdos, como los que llevaron a Bolivia a contraer empréstitos para construir ferrocarriles hacia Chile en beneficio de las empresas mineras nacionales y transnacionales en el siglo XIX, de un pasado dependiente. Esta situación resulta altamente contradictoria en el marco de un gobierno que accedió al poder bajo un programa de transformación profunda del modelo imperante.

La respuesta es que esto es parte de un proceso que tiene lugar en el último quinquenio. El MAS ha ido acercándose presurosamente a empresariado, exportadores e inversores extranjeros; estableciendo acuerdos y compromisos que se han convertido en la columna central de los planes gubernamentales de corto y mediano plazo. Este viraje devela un horizonte político reducido a un cúmulo de obras y proyectos principalmente de infraestructura de exportación que pretendidamente acrecentarán las divisas. La finalidad sería alcanzar el Vivir Bien, pero en una versión reconvertida en desarrollo integral.

Como ejemplo, el principal destino del crédito chino será la construcción de varias carreteras hacia el norte amazónico, un tren que abre camino hacia el yacimiento de hierro del Mutún y una hidroeléctrica para empezar la exportación de energía eléctrica. Así la llamada Agenda 2025, que es el principal justificativo para una nueva reelección presidencial post 2020, tiene entre sus pilares más y más extractivismo. El primer pilar de la Agenda, denominado Patria Grande e Industrial en la Era Satelital, pretende garantizar el desarrollo de la cadena productiva hidrocarburífera, así como de las diferentes formas de energía y sus fuentes, desarrollar, fomentar, promocionar y controlar la actividad minera y metalúrgica, fortalecer los corredores bioceánicos, etc. (Ministerio de Planificación del Desarrollo de Bolivia, 2014).

\footnotetext{
${ }^{3}$ Denominado Invertir en la nueva Bolivia se realizó el 26 de octubre de 2015. Un día después publicaron una separata denominada The new Bolivia (edición de 52 páginas) al parecer financiada por el gobierno, empresas estatales y privadas bolivianas.
} 
¿Qué sentido puede tener insistir en la exportación de materias primas en un contexto de baja de precios internacionales? Históricamente solo los sectores conservadores apostaron a incrementar los volúmenes exportados para compensar la baja de precios y solo ellos promovieron mayores subvenciones como las que ahora les promete Evo Morales a las petroleras extranjeras con un Fondo de Incentivo de 3.556 millones de dólares (Reuters, 2015).

Al igual que ocurre en Ecuador bajo Rafael Correa, el MAS de Bolivia se encuentra actualmente inmerso en una tesitura de aumento de las contradicciones entre su actual accionar gubernamental y el programa original con el que accedió al poder en 2006. Podríamos nombrar las iniciales medidas de su primer mandato, que luego fueron vistas por las autoridades como frenos al desarrollo, o que van siendo reemplazadas por medidas abiertamente contrarias. Es el caso de la exportación de gas, a la que tenazmente se opusieron movimientos sociales de El Alto en 2003. Bajo las políticas de Sánchez de Lozada, Bolivia empezó exportando el 46\% de lo extraído; en el año 2014, el 96,73\% de lo extraído fue exportado. Esta tendencia se agrava si se observa que los volúmenes se incrementaron durante el gobierno del MAS en casi cinco veces. Bolivia se hizo más dependiente de las exportaciones durante un gobierno que teóricamente debía recuperar la soberanía nacional.

En su búsqueda de mercados e inversiones, el gobierno de Morales confronta con múltiples sectores sociales, entre otros, los pueblos indígenas que ven comprometido su futuro por este embate extractivista. Este es el origen de una de las rupturas con el gobierno.

Ese mismo gobierno que promoviera el Estado Plurinacional en la Asamblea Constituyente (2006-2009), arremete poco tiempo después contra los derechos de los pueblos indígenas. Reprime duramente sus pacíficas movilizaciones (como ocurriera en octubre de 2011 en Chaparina o recientemente en el Chaco) y desentierra todo tipo de descalificaciones coloniales y racistas contra sus legítimas demandas. Las rémoras del Estado colonial remozan en un funcionariado del Estado Plurinacional que trata a las comunidades indígenas de incivilizadas y de obstáculos para el desarrollo del país.

Otros sectores sociales que, muchas veces, solamente le demandan participación en las decisiones que afectarán su futuro, son tratados como opositores y derechistas con los que no dialogan. La represión a la disidencia social aleja al gobierno de las reivindicaciones abanderadas en la Asamblea Constituyente de mayor control social al funcionariado público. ¿No era acaso uno de los fines de este proceso el radicalizar la democracia, trascender la democracia representativa $y$ transitar hacia formas participativa, directa y comunitaria?

Este desgaste cada vez más difícil de compensar, ocultar o disimular necesita además del control de medios de comunicación, de estrategias de control 
y/o de la eliminación de voces críticas y de la implementación de impresionantes campañas publicitarias. Para mantener "congelada» esa primera imagen de cambio y transformación se apela a un gigantesco y millonario despliegue publicitario: el gobierno destina 1 millón de Bs. diarios a propagandizarse (ERBOL, 2015).

Rafael Archondo analiza acertadamente el papel de la incesante publicidad gubernamental en esta puesta en escena: mostrar esas primeras e iniciales medidas - concretamente la nacionalización parcial de los hidrocarburos - como la «pócima» que resolvió todos los males y que terminó por desterrar del país a la derecha antinacional.

El fantasma de la derecha antinacional es sacudido ostentosamente en cada concentración humana. Ésta no existe ya en la vida real, pero cuán útil resulta a la hora de cohesionar a la sociedad en contra del pasado (Archondo, 2015).

Un repertorio muy útil asimismo para descalificar, estigmatizar, ilegalizar, e incluso judicializar cualquier cuestionamiento a sus políticas (sea de movimientos sociales, ONGs, periodistas o de su propia militancia) que sería perfectamente normal en un estado democrático que se precia de articularse al servicio de la sociedad. ¿No era acaso un principio básico del MAS el «mandar obedeciendo al pueblo»?

Este agotamiento del modelo económico planteado por gobiernos progresistas como el de Evo Morales, conlleva en el momento actual preocupantes transformaciones políticas reflejadas en una seria erosión de la democracia. Cuestionar al presidente o sus políticas extractivistas, supone formar parte de las fuerzas externas de derecha que conspiran contra la revolución. De este modo, proyectos gubernamentales como una carretera (pongamos el caso de la conocida propuesta de construir una ruta dentro del parque nacional conocido como Territorio Indígena y Parque Nacional Isiboro-Secure - TIPNIS-), una planta de energía nuclear, o la exploración petrolera en áreas protegidas, son tratadas como asuntos que comprometen la seguridad nacional y la oposición a las mismas es tildada de enemigo interno al que se ha de vencer. El vicepresidente, Álvaro García Linera, se ha esmerado en demostrar la peligrosidad de este nuevo terrorismo al que, insiste, no queda más que derrotar.

El alejamiento entre el MAS y los movimientos sociales —o de aquellos que pueden ser considerados como tales porque mantienen una agenda propia que no es dictada por las autoridades - es tan llamativo como el acercamiento de éste a sus antiguos sectores adversarios, como el latifundismo agropecuario de Santa Cruz. Este año, en acuerdo con ellos, se han dejado atrás los principales logros de la Constituyente, dando paso a una agenda agro-extractivista. Se 
ha levantado la prohibición a los transgénicos, que nuevamente pueden ser introducidos y producidos sin restricción; también se ha dado marcha atrás en la reforma agraria, evitando la verificación de la Función Social de la tierra por cinco ańos, lo que permitirá al latifundismo conservar su poder en las tierras bajas.

La banca también gana con el proceso de cambio, sus utilidades crecen año a año. Sus ganancias fueron de 43,6 MM de dólares en 2005, para trepar a los 288,3 MM de dólares en el 2014, a un ritmo promedio de $62 \%$ de crecimiento por año (Lazcano, 2015). El que los bancos acrecienten sus ganancias es tomado por las autoridades como un indicador de la "buena salud de la economia», como si éstos alguna vez hubieran compartido sus ganancias con la sociedad.

Con las transnacionales se ha seguido similar camino. Por ello, Repsol -la transnacional espańola que promueve un proyecto de exportación de gas a Estados Unidos, y a la que se puede responsabilizar de los hechos que derivaron en la Guerra del Gas de 2003- formó parte de la delegación que acompañó a Evo a promocionar la nueva imagen de Bolivia en Nueva York.

\section{Propuestas y alternativas al modelo energético extractivista boliviano}

Empezaremos por describir a grandes rasgos el modelo energético vigente. La producción de energía primaria está anclada en energía de origen fósil: 93\% (gas natural y petróleo) (Ministerio de Hidrocarburos y Energía de Bolivia, 2015). A pesar de la promesa del MAS de cambiar la matriz energética, en la última década, la mayor parte de la energía producida es fósil y además es exportada como se observa en el gráfico 1.

La profundización del modelo energético extractivista se apoyó en dos presupuestos. El primero tiene que ver con una extendida creencia, muy arraigada entre las autoridades sectoriales, que existen abundantes y casi inagotables recursos hidrocarburíferos que se encuentran dormidos en territorios como el amazónico. Este presupuesto no toma en cuenta la larga historia petrolera del país de cerca de 120 años. Tiempo en el que se ha desarrollado una extensa y muy costosa campaña exploratoria que ha comprometido a gran parte de las ecorregiones con potencial hidrocarburífero ${ }^{4}$. En los últimos años no se han descubierto importantes yacimientos petrolíferos y, por el contrario, éstos se encuentran ya en fase de declinación y/o agotamiento. Si a esta situación añadimos que los cos-

\footnotetext{
${ }_{4}^{4}$ Producto de esta campańa entre 1923 y 2012 se han descubierto 78 campos resultado de 303 estructuras perforadas y miles de kilómetros de exploración.
} 


\section{Gráfico 1}

Bolivia. Evolución de la oferta de energía por fuentes (en millones de BEP)

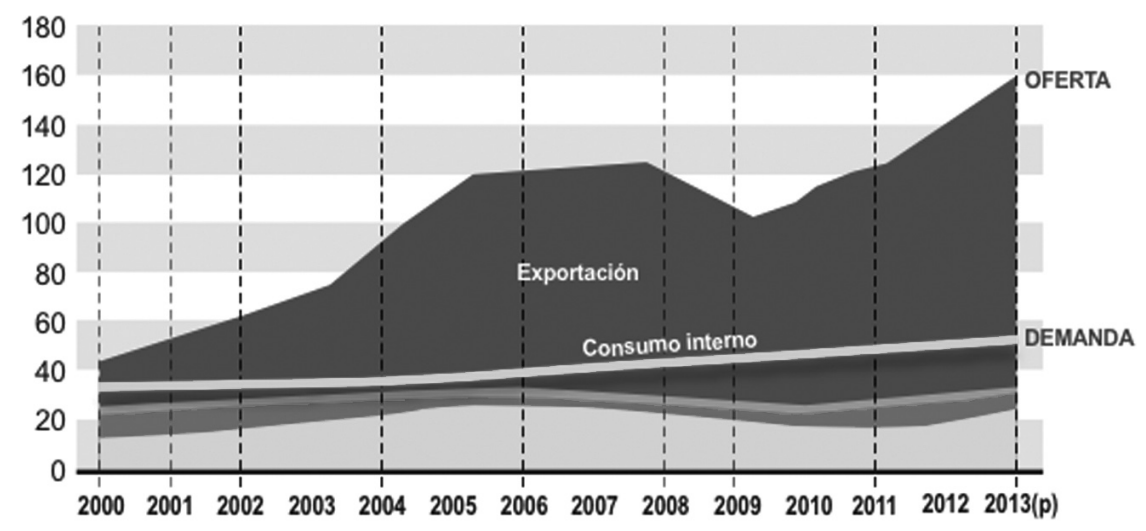

Petróleo Biomasa $\square$ Hidroenergía $\square$ Gas Natural

Consumo Interno

BEP: Barriles Equivalentes de Petróleo.

Fuente: Balance Energético Nacional. 2000-2013(p).

tos de extracción se incrementan en las zonas no tradicionales por la ausencia de infraestructura, tendremos como resultado que cada vez resulta más difícil y costosa la extracción de hidrocarburos, situación que se ha transformado en un límite sectorial.

El segundo presupuesto es de orden financiero y se resume en el mito de la dependencia de las exportaciones. Consiste en considerar que, sin las exportaciones, Bolivia se muere ${ }^{5}$. Y que cualquier alternativa a la exportación de materias primas haría inviable al Estado. En primer lugar, los ingresos por la exportación de recursos naturales, concretamente, gas natural (que es la principal materia prima exportada y que más aporta al fisco), es menor al $25 \%$ de los ingresos del Estado (ver gráfico 2).

Las recaudaciones por hidrocarburos no resultan vitales para las finanzas del estado boliviano, asimismo la capacidad efectiva de gasto de éste es bastante inferior a lo recaudado, en virtud a ello se ha acumulado una importante cantidad de recursos no ejecutados en los últimos años. El Ministerio de Econo-

5 La célebre consigna del ex presidente Víctor Paz Estensoro con la que se inauguraron las medidas neoliberales a mediados de los años 80 . 


\section{Gráfico 2}

Bolivia. Ingresos por hidrocarburos respecto al total de ingresos del Sistema Público no Financiero (1986-2012).

En \% y millones de Bs.

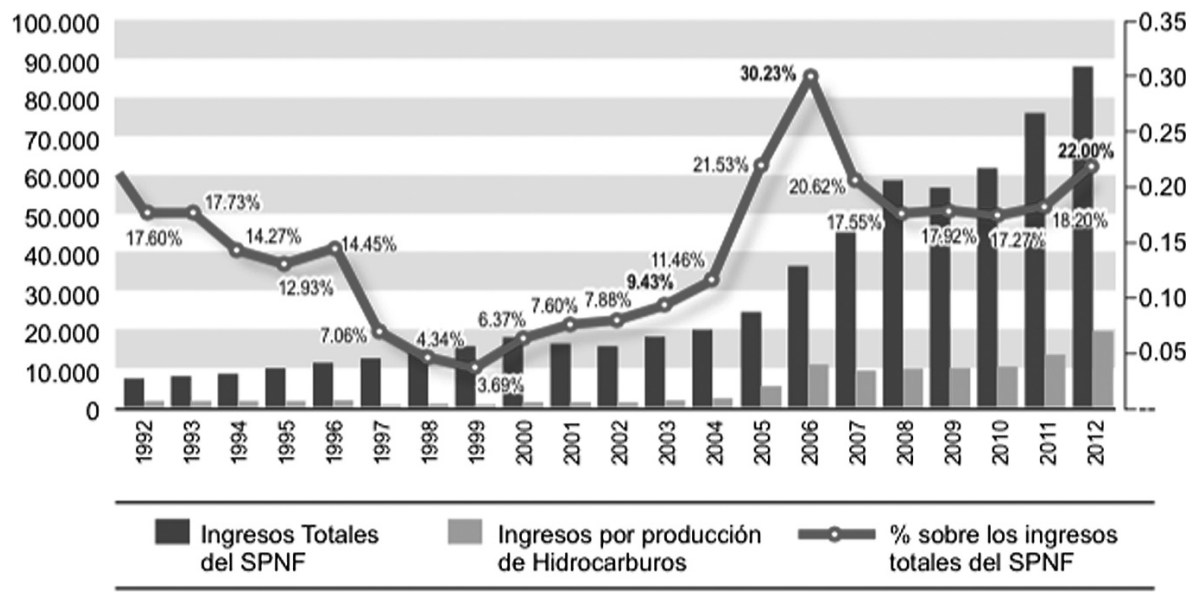

Fuente: Datos Hidrocarburiferos 1980-2012. CEDIB 2014.

mía y Finanzas estima que alrededor del $50 \%$ de lo transferido a niveles subnacionales no es ejecutado ${ }^{6}$. El problema es la visión obrista de la gestión pública que se resume en la construcción de obras vistosas pero de escasa utilidad pública que usualmente no responden a necesidades sociales o planes de largo plazo.

Si bien la mayor parte de la energía producida se exporta, a nivel interno se ha producido un significativo incremento del consumo de energía fósil, que además es importada debido a la carencia de petróleo. Esta tendencia se ha acrecentado por las constantes importaciones de automóviles particulares en desmedro del transporte público. El crecimiento del parque automotor en tan solo una década (2003-2013) es de $244 \%$ como se observa en el siguiente gráfico.

En base a este sucinto contexto nos permitimos esbozar algunas propuestas al modelo energético extractivista boliviano. Estas propuestas en varios frentes se desglosan de acuerdo con las prioridades de la agenda pública, a saber: a la agresiva exploración de hidrocarburos; el cambio de la matriz energética; la dependencia financiera de las exportaciones de gas natural y una agenda de derechos irrenunciables.

6 El saldo no ejecutado en 2014 fue de Bs. 16,3 mil millones de un total transferido de Bs. 26,8 mil millones. Ministerio de Economía y Finanzas Públicas. PGN 2015. 
Gráfico 3

Bolivia. Crecimiento del parque automotor (2003-2013)

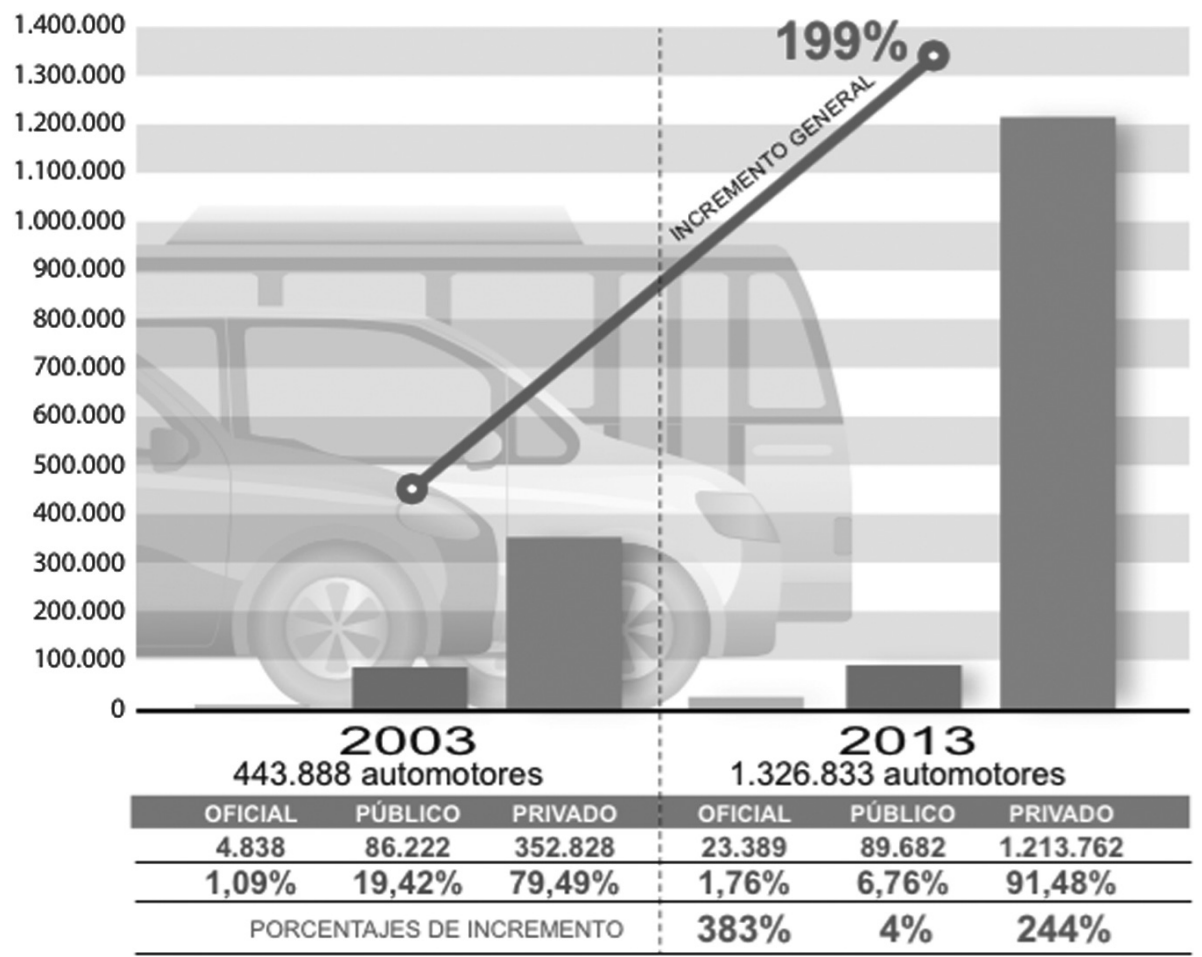

Fuente: elaboración propia en base a INE.

\subsection{Exploración-explotación hidrocarburos}

Diversos movimientos internacionales coinciden en que es necesario dejar el crudo bajo tierra ${ }^{7}$. Coinciden además en generar transiciones energéticas que posibiliten un cambio de matriz energética. Una línea de consenso tiene que ver con la moratoria petrolera que incluye medidas como: frenar créditos destinados a la apertura de nuevos yacimientos; eliminar subsidios a las empresas hidrocarburíferas; aplicar principios de intangibilidad en áreas protegidas, territorios indígenas y zonas ambientalmente frágiles o dedicadas a la seguridad alimentaria; y estimular la investigación y promoción de fuentes energéticas reno-

7 Declaración de Oil Watch abril 2015. http://www.oilwatch.org/dejar-el-crudo-en-elsubsuelo/680-dejar-los-combustibles-fosiles-debajo-de-la-tierra-una-declaracion-por-la-salud-de-lamadre-tierra 
vables $^{8}$. El principal problema interno a vencer es la orientación exportadora. Bolivia es un exportador neto de energía fósil. Un cambio fundamental es invertir esta orientación atendiendo a las necesidades energéticas internas. Dentro de esta línea, es posible plantear algunas medidas para el caso boliviano:
a) Abandonar exploración en Amazonía, Áreas Protegidas, SITIOS RAMSAR y Territorios Indígenas (moratoria petrolera) debido a los al- tos costos sociales, económicos y ambientales.
b) Renegociar exportación de gas con Argentina sobre gas natural empo- brecido en virtud a un acuerdo de integración energético/productivo. El convenio debe darse entre empresas estatales, sin intervención de nin- guna forma de empresas transnacionales.
c) Mantener en producción máx. 1 mega-campo dirigido a satisfacer la de- manda interna a largo plazo, en tanto se gestan medidas para el cambio de matriz energética.
d) Implementar una política de pasivos ambientales a escala nacional que considere el plan de cierre de campos menores y marginales.
e) Reorientar el Fondo de Incentivo Petrolero de la búsqueda de nuevos reservorios y la extracción a la conversión de la matriz energética y el fo- mento de actividades productivas sostenibles.
f) Compensaciones territoriales/productivas a pueblos y comunidades afectados por la actividad hidrocarburífera.
g) Restablecimiento de los Estudios de Evaluación de Impacto Ambiental en hidrocarburos. Innegociable cumplimiento Derechos de consulta li- bre, previa e informada.

\subsection{Energéticas}

Los cambios propuestos atañen a la política pública y a los actores estatales, aunque también a la sociedad misma, que como advertimos en el diagnóstico, ha incrementado el uso de energía fósil, como resultado del crecimiento del parque automotor en ausencia de sistemas de transporte público. Este aumento del consumo es claramente inequitativo y se sostiene debido al subsidio estatal. En esta línea es importante apuntar las siguientes acciones:

a) Eliminar progresivamente todos los subsidios energéticos a extractivismos: empezando por la soya y la minería.

b) Promover la inversión pública (con recursos nacionales, regionales y locales) en sistemas de transporte masivos eficientes. Paralelamente retomar la planificación urbana y políticas de acceso y mejora de vivienda social sin ampliaciones de las manchas urbanas.

${ }^{8}$ http://www.oilwatch.org/doc/campana/moratoria/moratoria-esp.pdf 
c) Resolver problema de internación de autos ilegales y la subvención de combustibles a vehículos particulares.

d) Incrementar la producción de energía aprovechando el potencial eólico, solar e hídrico de pequeña escala.

\subsection{Financieras}

Una de las mayores contradicciones del extractivismo boliviano se expresa en la inequitativa distribución del presupuesto público que, por el lado de los ingresos, castiga a los/as consumidores/as y trabajadores/as en beneficio de empresas mineras, soyeras, petroleras y exportadoras en general que se benefician de incentivos y exoneraciones tributarias. Debido a esto la ciudadanía soporta un gran peso fiscal, con el agravante de que se trata de un país marcadamente informal. Por el lado de los egresos, se favorece nuevamente al extractivismo al concentrar las inversiones públicas en infraestructura de exportación, hidrocarburos, hidroeléctricas, minería y agroindustria (Gandarillas, 2012). En este contexto, es fundamental atender la dimensión económica-financiera del extractivismo boliviano a través de las siguientes acciones:

a) Promover la participación social en un pacto fiscal. Dicho pacto deberá reorientar el sistema tributario, para lo cual, desde el punto de vista de los ingresos, se precisa:

- Establecer impuestos a la renta, patrimonio y sobreganancias empresariales.

— Disminuir la presión fiscal hacia los/as pobres y asalariados/as.

b) Priorizar la inversión pública hacia el fomento de producción interna, la atención a los derechos humanos y servicios básicos, transformando las reglas de la inversión pública, nuevamente es vital la participación y el control social en todo el ciclo de inversión:

- Concentrar las inversiones públicas en apoyo y fomento a la producción interna, la universalización de servicios básicos y transporte masivo. En esta área, promover inversiones concurrentes en políticas sociales: educación, salud, saneamiento básico, transporte público y vivienda social.

- Generar un fondo público con aportes de operadores y del Estado para saneamiento y conservación medioambiental.

\subsection{Derechos irrenunciables}

Dentro de este paquete de acciones urgentes, resulta fundamental el respeto por los derechos humanos y ambientales. Diversas organizaciones regionales plantean que el extractivismo está violentando los derechos humanos y 
de la naturaleza ${ }^{9}$. Esta arremetida violenta se expresa en normas y acciones estatales contra la libre asociación, la libertad de expresión y el derecho a la información y otros derechos colectivos como el derecho a la protesta y a la consulta libre previa e informada de los pueblos indígenas. Las acciones propuestas serían:

a) Respeto a los Derechos de la Naturaleza:

- La Autoridad Plurinacional de la Madre Tierra deberá reorganizarse para ejercer una efectiva defensa de las áreas de conservación, la restauración de los ecosistemas y un sistema plurinacional de monitoreo socio-ambiental nacional.

b) Derechos y libertades fundamentales:

- Respeto a derechos de libre expresión y asociación. La participación social es imprescindible para generar alternativas viables y sostenibles al extractivismo. Es imperativo que se abroguen las normas que limitan estos derechos y que, por el contrario, el estado, en todos sus niveles, promueva la más amplia participación social en la deliberación de los asuntos públicos.

c) Derechos colectivos de los pueblos indígenas:

— Dignificación de la territorialidad e identidad de los pueblos y naciones indígenas. Ejercicio de autogobierno.

d) Medidas para contener el poder de las empresas transnacionales:

- Imponer a la empresas transnacionales el cumplimiento de los más altos estándares sociales, laborales, ambientales.

- El obligado cumplimiento de normas de control financiero.

— Empleo de calidad. Sancionar la terciarización-subcontratación.

Quisiéramos que este listado de propuestas permitieran abrir un debate sobre la posibilidad de construir alternativas sostenibles y definitivas al extractivismo.

\section{Bibliografía}

AGENCIA DE NOTICIAS FIDES (2015): «Bolivia acepta analizar acuerdo comercial con la Unión Europea», $A N F, 11$ de noviembre.

\footnotetext{
9 Extrahección, es un concepto de Eduardo Gudynas que trata precisamente de esta relación entre los extractivismos y la violación de derechos. (2015:125-140).
} 
AGENCIA EFE (2014): «Ecuador y la UE firman acuerdo comercial que esperan aplicar en 2016", EFE, 12 de diciembre.

ARCHONDO, Rafael (2015): «En campaña permanente», La Razón, 14 de setiembre.

CAMPANINI, Oscar y GANDARILLAS, Marco A. (2015): "Agotamiento del progresismo en Bolivia: Profundización del extractivismo y erosión de la democracia», $A L A I$, 29 de octubre. http://www.alainet.org/es/articulo/173307

ERBOL (2015): «Ministerio de comunicación tiene cada día más de Bs. 1 millón para publicidad», ERBOL, 28 de agosto.

FARAH, Ivonne; VASAPOLLO, Luciano (coords.) (2011): Vivir Bien: ¿Paradigma no capitalista?, La Paz, CIDES-UMSA; SAPIENZA-Universidad de Roma; Fundación Xavier Albó.

GANDARILLAS, Marco A. (2012): «La orientación extractivista de la inversión pública», Bolpress, 30 de mayo. http://www.bolpress.com/art.php?Cod=2012053006

GANDARILLAS, Marco A. (2013): «Extractivismo y derechos laborales. Dilemas del caso boliviano", en HERNÁNDEZ, Juan; DE LA FUENTE, Mikel; DE VICENTE, Andrea; IRURZUN, Koldo (coords.): Empresas Transnacionales en América Latina. Análisis y propuestas del movimiento social y sindical, Bilbao, Hegoa, 218-232.

GUDYNAS, Eduardo (2011): «Más allá del nuevo extractivismo: Transiciones sostenibles y alternativas al desarrollo", en WANDERLEY, Fernanda (coord.) El desarrollo en cuestión: reflexiones desde América Latina, La Paz, CIDES-OXFAM.

GUDYNAS, Eduardo (2015): Extractivismos. Ecología, economía y politica de un modo de entender el desarrollo y la naturaleza, Cochabamba (Bolivia), CLAES-CEDIB.

GUDYNAS, Eduardo y ACOSTA, Alberto (2011): «El buen vivir más allá del desarrollo», Qué hacer 181: 70-81.

LAZCANO, Miguel (2015): «Ganancias de la banca crecen en 33\% y alcanzan a \$us 283 millones», La Razón, 14 de enero.

MINISTERIO DE HIDROCARBUROS Y ENERGÍA DE BOLIVIA (2010): Balance Energético Nacional. 2000-2010, La Paz, Estado Plurinacional de Bolivia.

MINISTERIO DE HIDROCARBUROS Y ENERGÍA DE BOLIVIA (2015): Balance Energético Nacional (2010-2015), La Paz, Estado Plurinacional de Bolivia.

MINISTERIO DE PLANIFICACIÓN DEL DESARROLLO (2014): Directrices de planificación de mediano y largo plazo hacia la agenda patriótica 2025, La Paz, Estado Plurinacional de Bolivia.

REUTERS (2015): «Bolivia creará fondo de US\$ 3,556 millones para incentivar inversiones extranjeras en hidrocarburos», Reuters, 21 de agosto. 